\title{
COZMO - A New Lightweight Stream Cipher
}

\author{
Rhea Bonnerji ${ }^{0000-0002-5825-8800}$, Simanta Sarkar 0000-0002-4210-2764, \\ Krishnendu Rarhi ${ }^{0000-0002-5794-215 X}$, Abhishek Bhattacharya \\ School of Information Technology, Institute of Engineering \& \\ Management, Kolkata
}

\begin{abstract}
This paper deals with the merger of the two lightweight stream ciphers - A5/1 and Trivium. The idea is to make the key stream generation more secure and to remove the attacks of the individual algorithms. The bits generated by the Trivium cipher (output) will act as the input of the A5/1 cipher. The registers used in the A5/1 cipher will be filled by the output bits of the Trivium cipher. The three registers will then be connected to generate an output which will be our required key stream.
\end{abstract}

Keywords: Lightweight stream cipher, A5/1, Trivium

\section{Introduction}

Cryptography is very important in today's times as we want confidential information to remain confidential. We don't want people other than the intended receivers to be able to access our information and if accessed to be able to be able to understand it. Cryptography is the method of converting information to a non-understandable form and then being able to convert it to the understandable form by the recipient. Lightweight stream ciphers are used to reach high levels of security using only a small computing power. Stream encryption is the encryption of each letter one by one followed by the changing the encryption key after each letter. Lightweight stream ciphers have the advantage of having low cost hardware implementations as they have high throughput and low complexity. The idea is to come up with a lightweight stream cipher algorithm using existing cipher algorithms that is secure to the attacks of the originally used cipher algorithms. Also, to generate a more secure keystream. Here, we are using Trivium and A5/1 algorithm and making changes to suit our needs. This paper has results of statistical tests and proves that our algorithm can be considered to be strong.

\subsection{Understanding the working of Trivium:}

Trivium generates up to $2^{64}$ bits of key stream from an 80 bit secret key and an 80 bit initialization vector (IV). First the internal state of the cipher is initialised using the key and the IV. Then the state is updated repeatedly to generate the key stream bits. It consists of 3 interconnected non-linear feedback shift registers. The length of the registers are 93, 84 and 111 bits, respectively. Initialisation requires 1152 steps of the clocking before key stream is generated. There are XOR gates to XOR the ouputs we get from the various registers which is again fed back to the first one. The 91 and 92 bits are ANDed and used to give the feedback to the $94^{\text {th }}$ bit. The 175 and 176 bits are ANDed and used to give the feedback to the $178^{\text {th }}$ bit. The 
286 and 287 bits are ANDed and used to give feedback to the $288^{\text {th }}$ bit. And the $288^{\text {th }}$ bit is giving back the feedback to the $1^{\text {st }}$ bit. [1]

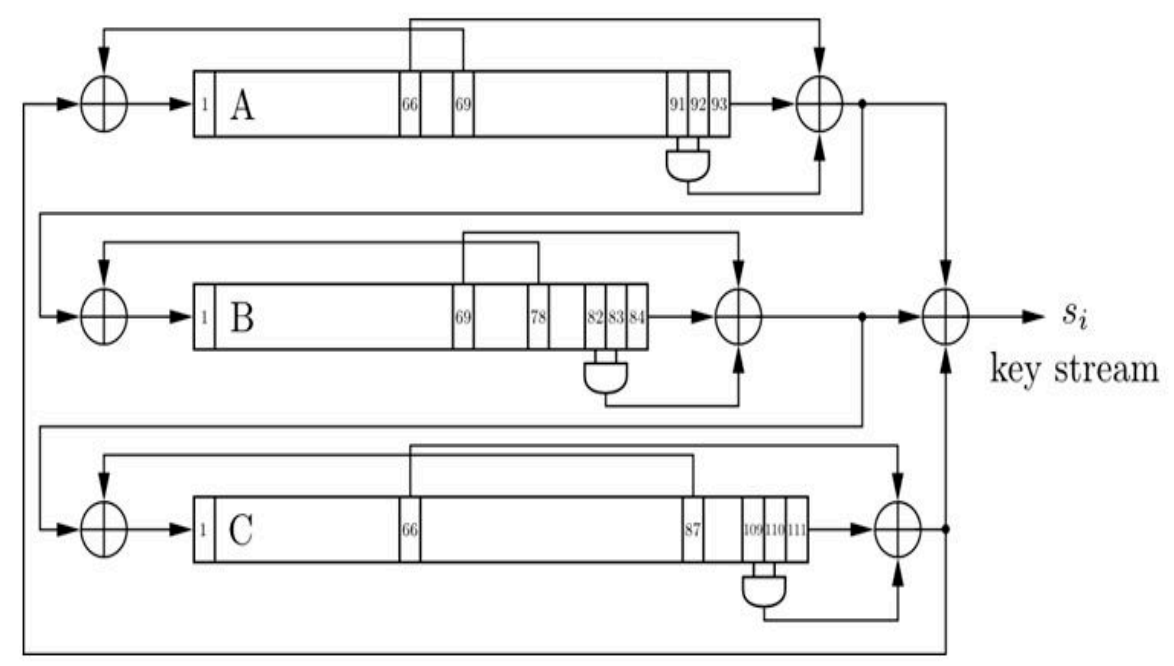

Fig. 1. Trivium

\section{Mathematical structure}

If we denote the internal state bits of the Trivium Model algorithm at time $t$ as

$\mathrm{z}(\mathrm{t})=\left(\mathrm{z} 1(\mathrm{t}), \mathrm{z} 2(\mathrm{t}), \ldots, \mathrm{z} 3_{\mathrm{n} 3}(\mathrm{t})\right)$, then the internal bits from time $\mathrm{t}$ to time $\mathrm{t}+1$ can be expressed as follows

$$
\mathrm{z}(\mathrm{t}+1)=\mathrm{A} \cdot \mathrm{z}(\mathrm{t})+\mathrm{b}(\mathrm{t})
$$

Where $A=\left(a_{i j}\right) 3_{n 3} 3_{n 3}$ is the state-transition matrix of the algorithm with size $3_{\mathrm{n} 3} \times 3_{\mathrm{n} 3}$

$$
a_{i j}= \begin{cases}1, & i=1, j=3 u_{2}, 3 u_{5}, 3 n_{2} \\ 1, & i=3 n_{1}+1, j=3 u_{1}, 3 u_{4} \\ 1, & i=3 n_{2}+1, j=3 u_{3}, 3 u_{6} \\ 1, & 1<i<=3 n_{3}, j=I-1 \\ 0, & \text { otherwise }\end{cases}
$$

$\mathrm{b}(\mathrm{t})=\left(\mathrm{b}_{\mathrm{i}}(\mathrm{t})\right) 3_{\mathrm{n} 3}$ is the nonlinear segment of the algorithm, which is treated as the vectors of bits

$$
b_{i}(t)= \begin{cases}z 3_{n 3-2}(t) \cdot z 3_{n 3-1}(t), & i=1 \\ z 3_{n 1-2}(t) \cdot z 3_{n 1-1}(t), & i=3 n_{1}+1 \\ z 3_{n 2-2}(t) \cdot z 3_{n 2-1}(t), & i=3 n_{2}+1 \\ 0, & \text { otherwise }\end{cases}
$$




\subsection{Understanding the working of A5/1:}

A5/1 uses 3 shift registers of 19, 22 and 23 bits respectively. We have a 64 bit key and we'll load it in the three registers. Then we'll do some process to generate as many bits as we want to and we'll use those bits as the key stream and XOR it with the plain text to encrypt and the cipher text to decrypt. There is a majority vote function which will take 3 bits and find the majority of them. The three shift registers are loaded with the 64 bit key. There are three special positions in the registers $-8,10$ and 10 respectively. Take the majority of these three bits and check which of the registers are in majority. If register 1 is in majority then take the $13^{\text {th }}, 16^{\text {th }}$, $17^{\text {th }}$ and $18^{\text {th }}$ bit and XOR them. If register 2 is in majority then XOR the bits in the $20^{\text {th }}$ and $21^{\text {st }}$ position. If register 3 is in majority then XOR the bits in the $7^{\text {th }}, 20^{\text {th }}, 21^{\text {st }}$ and $22^{\text {nd }}$ position. Only shift the XORed bits to the first position of the registers belonging in majority. The register which is not in majority will remain untouched. Then we have to XOR the bits in the last position of the registers and that is our required key stream bit. Repeating this process over and over again will give us our key stream. [2]

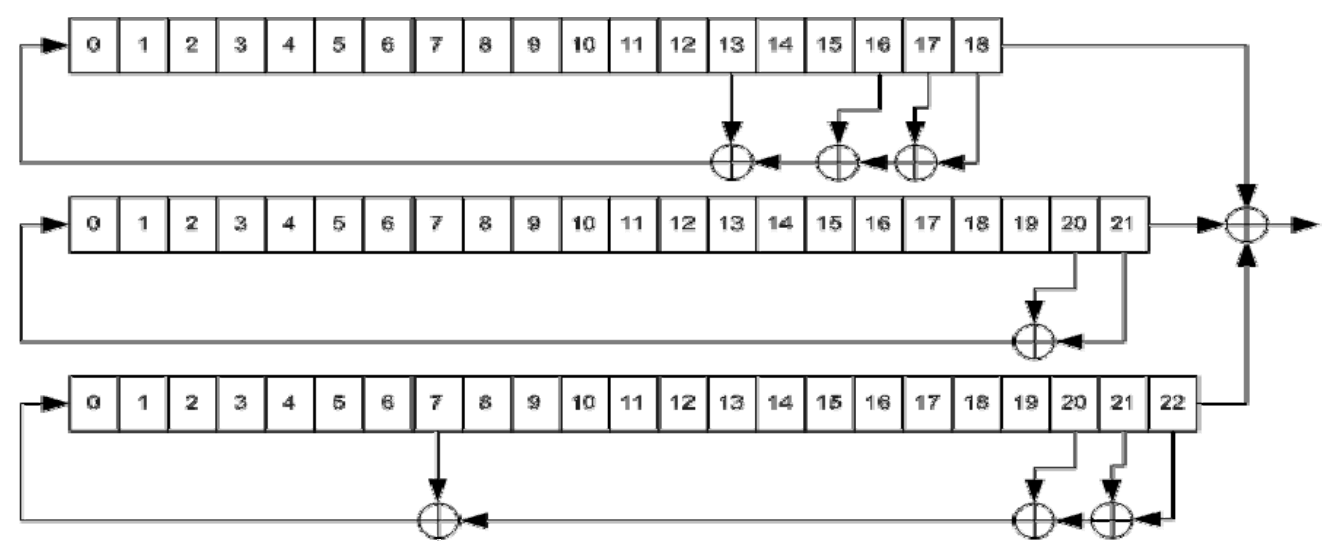

Fig. 2. A5/1

\section{Proposed Methodology}

The idea is to make the key stream even more secure by merging the two algorithms together. The Trivium cipher is initialised with a key and initialization vector and the A5/1 cipher is initialised with all 0's. First we will be generating a key stream using the Trivium algorithm. The generated key stream bits will be used as the input for the A5/1 cipher. There will be a clocking of $1216(1152+64)$ cycles before the key stream starts getting generated. Let the three registers be called register A, B and C respectively. First we XOR the selected position bits in all the registers. For the first register $\mathrm{A}$, the selected positions are the $13^{\text {th }}, 16^{\text {th }}$ and $17^{\text {th }}$ and $18^{\text {th }}$ bits. For the second register B, the selected positions are the $20^{\text {th }}$ and $21^{\text {st }}$ bits. For the third 
register $\mathrm{C}$, the selected positions are the $7^{\text {th }}, 20^{\text {th }}, 21^{\text {st }}$ and $22^{\text {nd }}$ bits. Next we check the majority function and find the two registers that are in majority. Next, we shift the bit in the last position to the first position by using right shift by one place only for the registers in majority. Once that is done, we will use the XOR bits. If register A is in majority and B isn't then discard the XORed bit from register A. Since register C is in majority, the XORed bit of register B will replace the bit in the first position of register C. Register A which is also in majority will have its first bit replaced by the XORed bit of the register $\mathrm{C}$ which is XORed with the bit generated by Trivium. The final key stream is the XOR of the three bits in the last positions of the three registers.

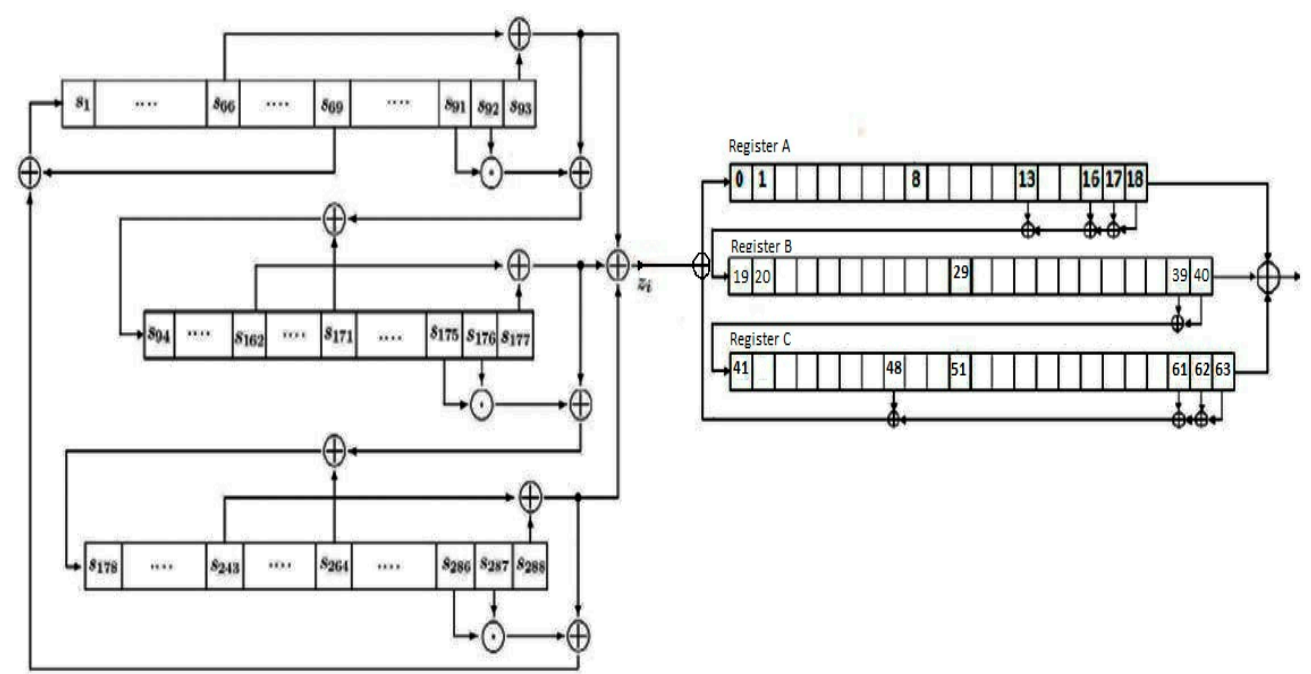

Fig. 3. COZMO

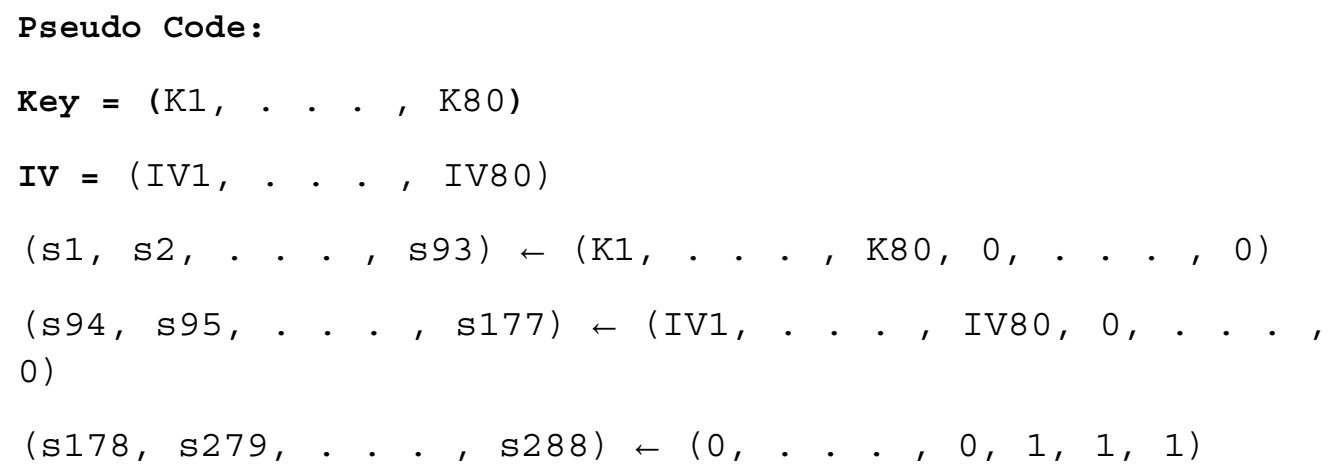

Register A:

$(r 0, r 2, . . ., r 18) \leftarrow(0,0, . . ., 0)$

Register B:

$(r 19, r 20, . . ., r 40) \leftarrow(0,0, . ., 0)$

Register C:

$(r 41, r 43, . . ., r 63) \leftarrow(0,0, . . ., 0)$ 


\section{Operations :}

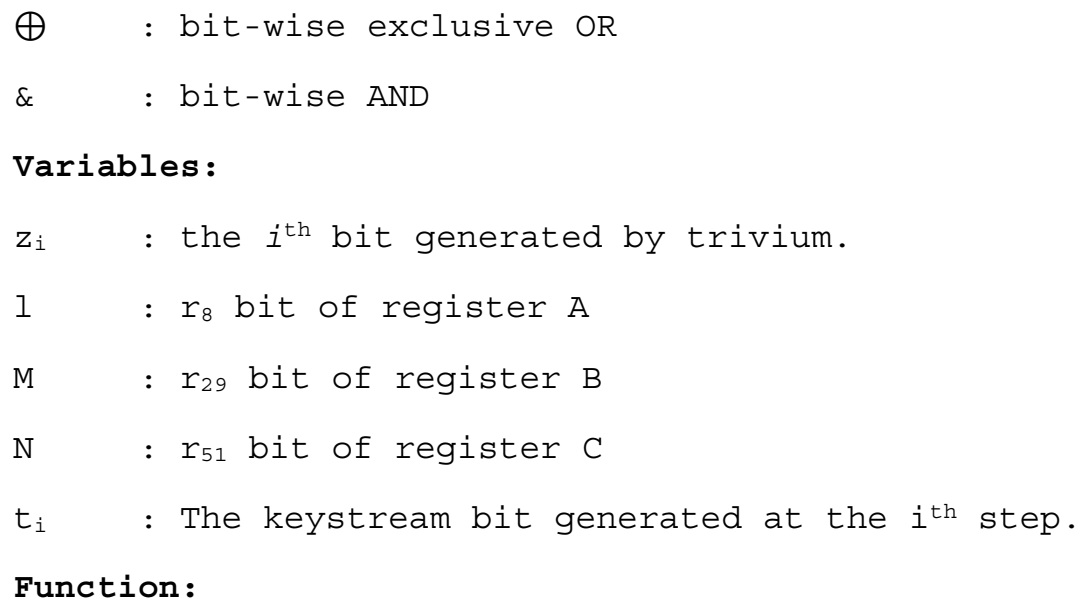

\section{Attacks:}

Correlation attack:

This attack is usually used on ciphers whose output is a combination of LFSRs and Boolean functions. This is possible when there is correlation between bit generated by the combination of bits used in the Boolean function and the output bit of the LFSRs. Our algorithm, however, has been tested and is secure from the correlation attack. 


\section{Results and Discussion}

As we know statistical analysis is a very important analysis for determining the acceptance or rejection of the hypothesis, the following tests were conducted to test the randomness property of the key bit generator. We performed basic statistical tests like frequency, longest run of one's in a block, linear complexity, entropy, etc. on our hypothesis. The result of the tests are shown in the tables below.

\begin{tabular}{|c|c|c|}
\hline Statistical Test & $\mathbf{p}$ - value & Success/failure \\
\hline Frequency & 0.534146 & Success \\
\hline Cumulative Sums & $\mathrm{P} 1-0.122325$ & Success \\
& $\mathrm{P} 2-0.350485$ & Success \\
\hline Approximate Entropy & 0.004301 & Success \\
\hline Linear Complexity & 0.213309 & Success \\
\hline Serial & $\mathrm{P} 1-0.004301$ & \\
\hline Longest Run of Ones & 0.350485 & Success \\
\hline Runs & 0.911413 & Success \\
\hline
\end{tabular}

Table 1: The Results of Statistical Analysis for A5/1

\begin{tabular}{|c|c|c|}
\hline Statistical Test & $\mathbf{p}$ - value & Success/failure \\
\hline Frequency & 0.534146 & Success \\
\hline Cumulative Sums & $\mathrm{P} 1-0.213309$ & Success \\
& $\mathrm{P} 2-0.350485$ & \\
\hline Approximate Entropy & 0.534146 & Success \\
\hline Linear Complexity & 0.035174 & Success \\
\hline Serial & $\mathrm{P} 1-0.911413$ & Success \\
& $\mathrm{P} 2-0.534146$ & \\
\hline Longest Run of Ones & 0.739918 & Success \\
\hline Runs & 0.350485 & Success \\
\hline
\end{tabular}

Table 2: The Results of Statistical Analysis for Trivium

\begin{tabular}{|c|c|c|}
\hline Statistical Test & p- value & Success/failure \\
\hline Frequency & 0.911413 & Success \\
\hline Cumulative Sums & P1-0.991468 & Success \\
& P2-0.739918 & \\
\hline Approximate Entropy & 0.350485 & Success \\
\hline Linear Complexity & 0.066882 & Success \\
\hline Serial & P1-0.213309 & Success \\
& P2-0.534146 & \\
\hline
\end{tabular}




\begin{tabular}{|c|c|c|}
\hline Longest Run of Ones & 0.534146 & Success \\
\hline Runs & 0.122325 & Success \\
\hline
\end{tabular}

Table 3: The Results of Statistical Analysis for COZMO

\section{Conclusion}

We wanted to propose a new lightweight stream cipher because of their higher speed, efficiency and its easy implementations on applications which require plain texts of unknown length. In this paper we merged and made some changes to the algorithms of two already existing lightweight stream ciphers - Trivium and A5/1 and proposed a new lightweight stream cipher with good key randomness as key randomness is a major issue is stream ciphers. The generated key stream is more secure compared to the key streams generated by the individual ciphers. Trivium is already known to be a pretty secure cipher. Any modifications made on it naturally increase the chances to the generated key stream to be more secure. Our algorithm also produces sequences which are stronger in statistical properties. The implementation of this algorithm is also very feasible and easy to put to use.

\section{References}

1. C. De Canni ere and B. Preneel, "Trivium - A Stream Cipher Construction Inspired by Block Cipher Design Principles,” 2006/021. (2006)

2. Timo Gendrullis, Martin Novotny, and Andy Rupp, “A Real-World Attack Breaking A5/1 within Hours" /2008/147 , (2008).

3. Diffie, W. y M.E.Hellman. "New directions in cryptography", IEEE Transactions on Information Theory 22 (1976), pp. 644-654.

4. Raddum, H.“Cryptanalytic Results on Trivium”, eSTREAM, ECRYPT Stream Cipher Project, Report 2006/039, 2006.

5. De Canniere C and Preneel B. TRIVIUM specifications. eSTREAM, ECRYPT stream cipher project. Report no. 2005/030, April 2005

6. Fischer, W., Gammel, B.M., Kniffler, O., Velten, J.: Differential power analysis of stream ciphers. In: Abe, M. (ed.) CT-RSA 2007. LNCS, vol. 4377, pp. 257-270. Springer, Heidelberg (2006)

7. Lennart Diedrich, Patrick Jattke, Lulzim Murati, Matthias Senker, and Alexander Wiesmaier, "Comparisons of Lightweight Stream Ciphers: MICLEY 2.0, WG-8, Grain and Trivium"

8. Maximov, A. and Biryukov, A. "Two Trivial Attacks on Trivium", Selected Areas in Cryptography, Lecture Notes in Computer Science, Vol.4876, Springer, 2007.

9. Jia, Y., Yupu, H., Wang, F., Wang, H.: Correlation power analysis of Trivium. Secur. Commun. Netw. 5(5), 479-484 (2012)

10. Biham E and Dunkelman O. Cryptanalysis of the A5/1 GSM stream cipher. In: Roy B and Okamoto E (eds) Progress in cryptology INDOCRYPT 2000. Berlin: Springer, 2000, pp.43-51.

11. Maximov, A., T. Johansson, and S. Babbage, An Improved Correlation Attack on A5/1, in Selected Areas in Cryptography, H. Handschuh and M. Hasan, Editors. 2005, Springer Berlin / Heidelberg. p. 1-18. 
12. Meier, W. and O. Staffelbach, Nonlinearity Criteria for Cryptographic Functions, in Advances in Cryptology - EUROCRYPT '89, J.-J. Quisquater and J. Vandewalle, Editors. 1990, Springer Berlin / Heidelberg. p. 549-562.

13. Barkan, E., E. Biham, and N. Keller, Instant Ciphertext-Only Cryptanalysis of GSM Encrypted Communication. Journal of Cryptology, 2008. 21(3): p. 392-429.

14. Gendrullis, T., M. Novotný, and A. Rupp, A RealWorld Attack Breaking A5/1 within Hours, in Cryptographic Hardware and Embedded Systems - CHES 2008, E. Oswald and P. Rohatgi, Editors. 2008, Springer Berlin / Heidelberg. p. 266-282.

15. Barkan, E., E. Biham, and N. Keller, Instant Ciphertext-Only Cryptanalysis of GSM Encrypted Communication, in Advances in Cryptology - CRYPTO 2003. 2003, Springer Berlin / Heidelberg. p. 600-616.

16. J. Lano, N. Mentens, B. Preneel, and I. Verbauwhede, "Power Analysis of Synchronous Stream Ciphers with Resynchronization Mechanism," in ECRYPT Workshop, SASC - The State of the Art of Stream Ciphers, pp. 327-333, 2004. 